ISSN 1991-8631

Original Paper

http://indexmedicus.afro.who.int

\title{
Evaluation de la qualité microbiologique et physico-chimique des poissons capturés et commercialisés au port de pêche industrielle de Cotonou
}

\author{
G.R. DEGNON ${ }^{1}$, T.J. DOUGNON ${ }^{2 *}$, S. TOUSSOU $^{1}$ et S.Y. MIGAN ${ }^{1}$ \\ ${ }^{1}$ Université d'Abomey-Calavi, Ecole Polytechnique d'Abomey-Calavi, Département du Génie des \\ Technologies Alimentaires. Laboratoire d'Etude et de Recherche en Chimie Appliquée, Unité de \\ Recherche en Génie Enzymatique Alimentaire. 01 BP 526 Cotonou, Bénin. \\ ${ }^{2}$ Ecole Polytechnique d'Abomey-Calavi (EPAC), Département de Production et Santé Animales, \\ Laboratoire de Recherches en Biologie Appliquée (LARBA), Université d'Abomey-Calavi (UAC), \\ 01 BP 2009 Cotonou, Bénin. \\ *Auteur correspondant, E-mail : gnimabou2002@yahoo.fr
}

\section{RESUME}

La présente étude a consisté à évaluer la qualité microbiologique et physico-chimique des poissons produits et commercialisés au port de pêche industrielle de Cotonou. Les Germes Aérobie Mésophiles (GAM), les Coliformes thermo-tolérants, les Staphylocoques présumés pathogènes, les Anaérobies Sulfito-Réducteurs et les Salmonelles ont été recherchés selon la norme NF V08-010. Le pH et l'ABVT ont été déterminés dans les mêmes échantillons afin d'évaluer leur niveau de dégradation. Il est apparu que, la qualité bactériologique des poissons analysés n'est pas satisfaisante en comparaison à la norme par rapport à leur charge en coliformes. Ces analyses ont permis de constater que $24 \%$ des poissons sont contaminés par les Spores des ASR au niveau des bateaux et $48 \%$ à la halle des marées. En ce qui concerne la GAM, $100 \%$ des échantillons se trouvent dans la limite de tolérance $\left(5.10^{4}-5.10^{5}\right)$ UFC/g aussi bien au niveau des bateaux qu'à la halle des marées. Les teneurs en ABVT des poissons collectés à la halle des marées sont supérieures à celles obtenues au niveau des bateaux, avec $4 \%$ de non conformité. La teneur en ABVT des poissons prélevés au niveau des bateaux n'est pas significativement différente de celle des poissons prélevés au niveau de la halle des marées au seuil de $5 \%$, ce qui indique que le niveau de dégradation est satisfaisant. Cette étude a ainsi permis de montrer qu'un effort reste à faire quant à l'amélioration de la qualité sanitaire des poissons produits et commercialisés au Port de Pêche industrielle de Cotonou.

(C) 2012 International Formulae Group. All rights reserved.

Mots clés : Poissons, halle des marées, qualité microbiologique, qualité physico-chimique, état hygiénique, Bénin.

\section{INTRODUCTION}

La pêche joue un important rôle dans le développement socio-économique du Bénin en contribuant à environ 3\% du PIB (FAO, 2006). Le poisson constitue au Bénin, la source de protéine animale la plus importante dans l'alimentation de la population (Lalèyè, 1995). Environ les trois quarts de la production halieutique nationale sont consommés frais, le reste est fumé, séché, 
salé, ou frit avant d'être distribué sur les marchés intérieurs du pays (Gbaguidi et Fiogbé, 1998). Avec une valeur hautement nutritive, les poissons constituent un complément précieux dans les régimes alimentaires pauvres en protéines, vitamines et sels minéraux (FAO, 2006). Cependant, les poissons sont des denrées alimentaires très périssables, avec une vitesse d'altération relativement élevée après la pêche (Gram et al., 1987; Liston, 1992). En effet, entre 25-30 ${ }^{\circ} \mathrm{C}$ dans les pays tropicaux, les poissons s'altèrent en moins de 12 heures (FAO, 2006). Cependant, les bonnes pratiques de pêche et d'hygiène au débarquement, au niveau des mareyeurs ainsi qu'au niveau du matériel de production, permettent de prolonger la durée de conservation du poisson et de garantir sa qualité sanitaire. Cette disposition pourrait favoriser une amélioration de la qualité nutritionnelle, organoleptique et sanitaire des poissons frais.

Mais, de toutes les dispositions prises pour avoir une bonne qualité des poissons frais et propres à la consommation, les conditions de manipulation et de stockage des produits de pêche à la halle des marées du Port de pêche de Cotonou restent toujours inappropriées. Cette situation entraîne une contamination des bactéries pathogènes au niveau des poissons. Ces bactéries constituent les principales sources de contamination des poissons qui, crus ou insuffisamment traités, sont une voie épidémiologique importante de transmission des maladies microbiennes pouvant entraîner des cas de toxi-infections alimentaires (Sylla, 2000). Ces infections ont des conséquences particulièrement graves dans les pays en voie de développement et posent donc le problème de santé publique (Kaferstein, 1997). Au Maroc, 86\% des cas de toxi-infections alimentaires entre 2000 et 2004 sont d'origine bactérienne dont $10 \%$ des cas déclarés sont provoqués par les produits de pêche contaminés (Cohen et al., 2006).

Les conditions de production au port de pêche industrielle de Cotonou sont parfois inquiétantes et mettent en doute la qualité des poissons produits. Le présent travail vise à examiner la qualité microbiologique et physico-chimique des poissons débarqués et commercialisés à la halle des marées du port de pêche de Cotonou.

\section{MATERIEL ET METHODES Matériel}

Le matériel est constitué d'échantillons des espèces de poissons frais suivants : Pseudotolithus spp., Galeoides deacadactylus, Spyraena spp., Drepane africana, Alectis alexandrinus, Dasyatis margarita. Ces poissons ont été prélevés d'une part, au niveau des bateaux (échantillons $\mathrm{A}_{1}$ à $\mathrm{A}_{10}$ ) où ils sont débarqués et, d'autre part, au niveau de la halle des marées (échantillons $\mathrm{B}_{1}$ à $\mathrm{B}_{10}$ ) où ils sont vendus aux revendeuses. Les prélèvements ont été réalisés de manière aseptique dans des sacs de Stomacher et transportés au laboratoire à $4{ }^{\circ} \mathrm{C}$.

\section{Méthodes \\ Echantillonnage}

$\mathrm{Au}$ niveau de chaque bateau, 5 prélèvements ont été effectués au niveau des différents compartiments du bateau. Chaque prélèvement est constitué d'un lot de 10 poissons mélangés puis, un poisson a été prélevé de manière aléatoire et utilisé pour les analyses. Dans chaque bateau, 50 poissons ont été prélevés (5 lots de 10 poissons) puis un poisson par lot a été choisi, soit au total, 5 poissons ont été analysés. Il en est de même pour les prélèvements effectués au niveau de la halle des marées.

\section{Analyses \\ Analyses microbiologiques \\ Les analyses microbiologiques ont porté sur la recherche de Salmonelles et le dénombrement des Germes Aérobies Mésophiles (GAM), des Staphylocoques présumés pathogènes, des spores de microorganismes Anaérobies Sulfito- Réducteurs (ASR), les Coliformes thermo- tolérants (totaux et fécaux) et la détection des salmonelles. Pour réaliser ces analyses, il a été réalisé une suspension mère suivie d'une dilution décimale selon la norme NF V08-010}


(AFNOR, 1996) et du dénombrement de chaque germe précité par la méthode des dilutions (Tableau 1).

Analyses physico-chimiques

Le pH (AOAC, 1995) et l'Azote Basique Volatil Total (ABVT) (Pearson, 1976) ont été déterminés sur chaque échantillon de poisson.

\section{Analyses statistiques}

Les résultats des analyses ont été synthétisés et traités grâce au tableur Microsoft Excel 2007. Le logiciel SPSS v 16.0 a servi à analyser les données traitées par le calcul des fréquences, la comparaison des moyennes et l'analyse de la variance (ANOVA) par le test de Student NewmanKeuls.

\section{RESULTATS}

Evaluation microbiologique des poissons analysés

Les principaux micro-organismes dénombrés sur 100 échantillons de poissons prélevés (sur les deux lieux de prélèvement) sont consignés dans les Tableaux 2 et 3 . Ces résultats indiquent que les niveaux de contamination des poissons varient en fonction de la flore recherchée et du lieu de prélèvement. D'une manière générale, on note l'absence totale des salmonelles dans tous les échantillons de poissons analysés. Ce qui est conforme aux normes en vigueur qui recommandent une absence de salmonelles dans $25 \mathrm{~g}$ de produit.

Les Staphylocoques présumées pathogènes sont également absents dans les
100 échantillons analysés. Cela suppose aussi l'absence des Staphylococcus aureus, la seule espèce qui intéresse la bactériologie alimentaire en raison de son aptitude à libérer des entérotoxines qui sont à l'origine de graves intoxications alimentaires. Quant aux Germes Aérobies Mésophiles (GAM), au niveau des bateaux, tous les échantillons ont un niveau de contamination conforme à la norme dont la limite est $\left[5.10^{4} \mathrm{UFC} / \mathrm{g}\right.$, $\left.5.10^{5} \mathrm{UFC} / \mathrm{g}\right]$. Au niveau de la halle des marées, on a noté une augmentation du niveau de contamination. Environ $12 \%$ $(6 / 50)$ sont conformes à la norme (inférieur à $\left.\mathrm{M}=5.10^{5} \mathrm{UFC} / \mathrm{g}\right)$. Tous les échantillons de poissons analysés ont un taux élevé de Coliformes thermo-tolérants. Par ailleurs, on note que le niveau de contamination des coliformes est plus élevé au niveau de la halle des marées qu'au niveau des bateaux. Quant aux anaérobies sulfito-réducteurs (ASR), elles n'ont été révélées que dans les $24 \%$ des échantillons prélevés (12/50), au niveau des bateaux et dans les $48 \%$ des échantillons prélevés (24/50), au niveau de la halle des marées. Pour chaque germe testé, les analyses statistiques ont révélé qu'il n'y a pas de différence significative au seuil de 5\% entre les échantillons de poissons prélevés au niveau des bateaux et ceux prélevés au niveau de la halle des marées.

\section{Caractéristiques physico-chimiques des poissons analysés}

Les valeurs de $\mathrm{PH}$ et l'ABVT sont consignés dans le Tableau 4.

Tableau 1 : Germes recherchés et températures d'incubation.

\begin{tabular}{lc}
\hline Germes recherchés & Températures d'incubation \\
\hline Germes Aérobies Mésophiles (GAM) & $30^{\circ} \mathrm{C}$ pendant $72 \mathrm{H}$ \\
Coliformes totaux (CT) & $37^{\circ} \mathrm{C}$ pendant $24 \mathrm{H}$ \\
Coliformes fécaux (CF) & $44^{\circ} \mathrm{C}$ pendant $24 \mathrm{H}$ \\
Anaérobies-Sulfito Réducteurs (ASR) & $37^{\circ} \mathrm{C}$ pendant $48 \mathrm{H}$ \\
Staphylocoques & $37^{\circ} \mathrm{C}$ pendant $24 \mathrm{H}$ \\
Salmonelles & EPT à $37{ }^{\circ} \mathrm{C}$ pendant $18 \mathrm{H}$ \\
& RV à $42^{\circ} \mathrm{C}$ pendant $24 \mathrm{H}$ \\
& Hektoen à $37{ }^{\circ} \mathrm{C}$ pendant $24 \mathrm{H}$ \\
\hline
\end{tabular}


Tableau 2 : Flore microbienne moyenne dénombrée dans les échantillons de poissons prélevés au niveau des bateaux.

\begin{tabular}{|c|c|c|c|c|}
\hline \multirow[t]{2}{*}{ Bateaux } & \multirow{2}{*}{$\begin{array}{l}\text { GAM en UFC/g de } \\
\text { Produits }\end{array}$} & \multicolumn{2}{|c|}{ Coliformes en UFC/g de produits } & \multirow[t]{2}{*}{ ASR en UFC/g de produits } \\
\hline & & Totaux & Fécaux & \\
\hline $\mathrm{A}_{1}(\mathrm{n}=5)$ & $5,00 \cdot 10^{4} \pm 2,48 \cdot 10^{4} \mathrm{a}$ & $2,0.10^{4} \pm 1,2.10^{4} \mathrm{a}^{2}$ & $5,00 \cdot 10^{2} \pm 0,68 \cdot 10^{2} \mathrm{~b}$ & Absence \\
\hline$A_{2}(n=5)$ & $9,00 \cdot 10^{4} \pm 3,06 \cdot 10^{4} \mathrm{a}$ & $7,7 \cdot 10^{3} \pm 0,6 \cdot 10^{3} \mathrm{a}^{2}$ & $1,00 \cdot 10^{2} \pm 0,91 \cdot 10^{2} \mathrm{~b}$ & Présence dans 01 échantillon \\
\hline $\mathrm{A}_{3}(\mathrm{n}=5)$ & $4,00 \cdot 10^{4} \pm 1,11 \cdot 10^{4} \mathrm{a}$ & $2,0.10^{4} \pm 1,5.10^{4} \mathrm{a}^{2}$ & $2,00 \cdot 10^{2} \pm 1,25 \cdot 10^{2} \mathrm{~b}$ & Présence dans 01 échantillon \\
\hline $\mathrm{A}_{4}(\mathrm{n}=5)$ & $6,00 \cdot 10^{4} \pm 2,64 \cdot 10^{4} a$ & $2,2 \cdot 10^{4} \pm 0,1 \cdot 10^{4} \mathrm{a}^{2}$ & $6,25 \cdot 10^{2} \pm 2,64 \cdot 10^{2} \mathrm{~b}$ & Absence \\
\hline$A_{5}(n=5)$ & $7,00 \cdot 10^{4} \pm 2,82 \cdot 10^{4} \mathrm{a}$ & $6,2 \cdot 10^{3} \pm 2,4 \cdot 10^{3} \mathrm{a}^{2}$ & $2,35.10^{2} \pm 1,64 \cdot 10^{2} \mathrm{~b}$ & Présence dans 03 échantillons \\
\hline $\mathrm{A}_{6}(\mathrm{n}=5)$ & $1,42.10^{5} \pm 1,20.10^{5} a$ & $5,1 \cdot 10^{4} \pm 0,4 \cdot 10^{4} \mathrm{a}^{2}$ & $3,15 \cdot 10^{2} \pm 0,6 \cdot 10^{2} b$ & Présence dans 01 échantillon \\
\hline$A_{7}(n=5)$ & $1,57 \cdot 10^{5} \pm 1,51.10^{5} \mathrm{a}$ & $2,4 \cdot 10^{4} \pm 1,4 \cdot 10^{4} \mathrm{a}^{2}$ & $1,00.10^{3} \pm 1,00.10^{2} \mathrm{~b}$ & Présence dans 02 échantillons \\
\hline$A_{8}(n=5)$ & $4,15 \cdot 10^{5} \pm 2,20.10^{4} \mathrm{a}$ & $8,0.10^{4} \pm 3,1.10^{4} \mathrm{a}^{2}$ & $1,45 \cdot 10^{2} \pm 0,04 \cdot 10^{2} \mathrm{~b}$ & Présence dans 03 échantillons \\
\hline$A_{9}(n=5)$ & $8,65 \cdot 10^{4} \pm 2,40 \cdot 10^{4} a$ & $7,7.10^{4} \pm 1,9.10^{4} \mathrm{a}^{2}$ & $7,15 \cdot 10^{2} \pm 3,4 \cdot 10^{2} \quad b$ & Absence \\
\hline $\mathrm{A}_{10}(\mathrm{n}=5)$ & $7,50 \cdot 10^{5} \pm 1,40.10^{4} a$ & $1,9 \cdot 10^{4} \pm 0,75 \cdot 10^{4} \mathrm{a}^{2}$ & $3,00 \cdot 10^{2} \pm 1,64 \cdot 10^{2} \mathrm{~b}$ & Présence dans 01 échantillon \\
\hline Normes & $\left(5.10^{4}-5.10^{5}\right)$ & $10^{3}$ & 0 & 2 \\
\hline
\end{tabular}


Tableau 3 : Flore microbienne moyenne dénombrée dans les échantillons de poissons prélevés au niveau de la halle des marées.

\begin{tabular}{lcccc}
\hline $\begin{array}{l}\text { Halle des } \\
\text { marées }\end{array}$ & $\begin{array}{c}\text { GAM en UFC/g de } \\
\text { produits }\end{array}$ & \multicolumn{2}{c}{ Coliformes en UFC/g de produits } & ASR en UFC/g de produits \\
\cline { 2 - 4 } & & Totaux & Fécaux & \\
\hline $\mathrm{B}_{1}(\mathrm{n}=5)$ & $2,56.10^{7} \pm 2,48.10^{7} \mathrm{a}$ & $3,00.10^{5} \pm 1,2.10^{4} \mathrm{a}^{2}$ & $8.10^{2} \pm 0,68.10^{2} \mathrm{~b}$ & Présence dans 01 échantillon \\
$\mathrm{B}_{2}(\mathrm{n}=5)$ & $9,00.10^{6} \pm 3,06.10^{6} \mathrm{a}$ & $2,70.10^{5} \pm 0,6.10^{3} \mathrm{a}^{2}$ & $7,25.10^{2} \pm 0,91.10^{2} \mathrm{~b}$ & Présent dans 02 échantillons \\
$\mathrm{B}_{3}(\mathrm{n}=5)$ & $4,00.10^{7} \pm 1,11.10^{7} \mathrm{a}$ & $9,00.10^{4} \pm 1,5.10^{4} \mathrm{a}^{2}$ & $8,00.10^{2} \pm 1,25.10^{2} \mathrm{~b}$ & Présent dans 02 échantillons \\
$\mathrm{B}_{4}(\mathrm{n}=5)$ & $2,60.10^{5} \pm 0,92.10^{5} \mathrm{a}$ & $3,00.10^{5} \pm 0,46.10^{5} \mathrm{a}^{2}$ & $1,00.10^{3} \pm 1,37.10^{3} \mathrm{~b}$ & Présent dans 02 échantillons \\
$\mathrm{B}_{5}(\mathrm{n}=5)$ & $8,00.10^{6} \pm 1,39.10^{6} \mathrm{a}$ & $8,10.10^{4} \pm 0,7.10^{4} \mathrm{a}^{2}$ & $9,00.10^{2} \pm 0,98.10^{2} \mathrm{~b}$ & Présent dans 04 échantillons \\
$\mathrm{B}_{6}(\mathrm{n}=5)$ & $3,3.10^{6} \pm 2,60.10^{6} \mathrm{a}$ & $1,90.10^{5} \pm 1,6.10^{5} \mathrm{a}^{2}$ & $1,10.10^{3} \pm 1,04.10^{3} \mathrm{~b}$ & Présent dans 02 échantillons \\
$\mathrm{B}_{7}(\mathrm{n}=5)$ & $2,53.10^{6} \pm 2,1.10^{6} \mathrm{a}$ & $3,90.10^{5} \pm 2,82.10^{4} \mathrm{a}^{2}$ & $7,25.10^{2} \pm 1,82.10^{2} \mathrm{~b}$ & Présent dans 03 échantillons \\
$\mathrm{B}_{8}(\mathrm{n}=5)$ & $1,32.10^{8} \pm 0,2210^{8} \mathrm{a}$ & $9,98.10^{4} \pm 1,4.10^{4} \mathrm{a}^{2}$ & $8,98.10^{2} \pm 0,98.10^{2} \mathrm{~b}$ & Présent dans 04 échantillons \\
$\mathrm{B}_{9}(\mathrm{n}=5)$ & $1,04.10^{6} \pm 0,42.10^{6} \mathrm{a}$ & $1,20.10^{5} \pm 3,4.10^{5} \mathrm{a}^{2}$ & $9,12.10^{2} \pm 1,39.10^{2} \mathrm{~b}$ & Présent dans 02 échantillons \\
$\mathrm{B}_{10}(\mathrm{n}=5)$ & $3,62.10^{7} \pm 2,79.10^{7} \mathrm{a}$ & $2,05.10^{5} \pm 0,92.10^{5} \mathrm{a}^{2}$ & $7,98.10^{2} \pm 1,38.10^{2} \mathrm{~b}$ & Présent dans 02 échantillons \\
Normes & $\left(5.10^{4}-5.10^{5}\right)$ & & 0 & 2 \\
\hline
\end{tabular}

Les chiffres portant la même lettre dans la même colonne ne sont pas significativement différents au seuil de 5\%. 
Tableau 4: $\mathrm{pH}$ et teneur en ABVT des échantillons de poissons analysés au niveau des bateaux et à la halle des marées.

\begin{tabular}{lccc}
\hline Lieu de prélèvement & Echantillons & pH & ABVT $(\mathbf{m g}$. N/100 g) \\
\hline & $\mathrm{A}_{1}(\mathrm{n}=5)$ & $6,25 \pm 0,30 \mathrm{a}$ & $18,67 \pm 0,76 \mathrm{~b}$ \\
$\mathrm{~A}_{2}(\mathrm{n}=5)$ & $6,43 \pm 0,32 \mathrm{a}$ & $15,96 \pm 0,76 \mathrm{~b}$ \\
$\mathrm{~A}_{3}(\mathrm{n}=5)$ & $6,21 \pm 0,05 \mathrm{a}$ & $14,43 \pm 0,52 \mathrm{~b}$ \\
$\mathrm{~A}_{4}(\mathrm{n}=5)$ & $6,12 \pm 0,11 \mathrm{a}$ & $19,01 \pm 0,26 \mathrm{~b}$ \\
& $\mathrm{~A}_{5}(\mathrm{n}=5)$ & $6,04 \pm 0,08 \mathrm{a}$ & $14,86 \pm 1,49 \mathrm{~b}$ \\
Bateaux & $\mathrm{A}_{6}(\mathrm{n}=5)$ & $6,60 \pm 0,12 \mathrm{a}$ & $15,00 \pm 1,08 \mathrm{~b}$ \\
& $\mathrm{~A}_{7}(\mathrm{n}=5)$ & $6,60 \pm 0,50 \mathrm{a}$ & $16,30 \pm 3,10 \mathrm{~b}$ \\
& $\mathrm{~A}_{8}(\mathrm{n}=5)$ & $6,60 \pm 0,17 \mathrm{a}$ & $13,96 \pm 2,18 \mathrm{~b}$ \\
& $\mathrm{~A}_{9}(\mathrm{n}=5)$ & $6,27 \pm 0,12 \mathrm{a}$ & $16,50 \pm 1,2 \mathrm{~b}$ \\
& $\mathrm{~A}_{10}(\mathrm{n}=5)$ & $6,17 \pm 0,19 \mathrm{a}$ & $17,24 \pm 1,31 \mathrm{~b}$ \\
\hline $\mathrm{B}_{1}(\mathrm{n}=5)$ & $6,79 \pm 0,17 \mathrm{a}$ & $20,72 \pm 0,26 \mathrm{~b}$ \\
$\mathrm{~B}_{2}(\mathrm{n}=5)$ & $6,91 \pm 0,08 \mathrm{a}$ & $18,86 \pm 1,14 \mathrm{~b}$ \\
$\mathrm{~B}_{3}(\mathrm{n}=5)$ & $6,66 \pm 0,11 \mathrm{a}$ & $16,58 \pm 1,49 \mathrm{~b}$ \\
$\mathrm{~B}_{4}(\mathrm{n}=5)$ & $6,90 \pm 0,32 \mathrm{a}$ & $21,20 \pm 0,74 \mathrm{~b}$ \\
$\mathrm{~B}_{5}(\mathrm{n}=5)$ & $6,60 \pm 0,12 \mathrm{a}$ & $20,03 \pm 1,01 \mathrm{~b}$ \\
$\mathrm{~B}_{6}(\mathrm{n}=5)$ & $6,88 \pm 0,08 \mathrm{a}$ & $22,72 \pm 0,90 \mathrm{~b}$ \\
$\mathrm{~B}_{7}(\mathrm{n}=5)$ & $7,00 \pm 0,40 \mathrm{a}$ & $18,04 \pm 0,39 \mathrm{~b}$ \\
$\mathrm{~B}_{8}(\mathrm{n}=5)$ & $6,84 \pm 0,09 \mathrm{a}$ & $19,40 \pm 1,50 \mathrm{~b}$ \\
$\mathrm{~B}_{9}(\mathrm{n}=5)$ & $6,67 \pm 0,31 \mathrm{a}$ & $18,75 \pm 2,80 \mathrm{~b}$ \\
$\mathrm{~B}_{10}(\mathrm{n}=5)$ & $6,77 \pm 0,40 \mathrm{a}$ & $24,24 \pm 0,12 \mathrm{~b}$ \\
\hline Les chiffres portant la même lettre dans $\mathrm{a}$ même colonne ne sont pas significativement différents au seuil de 5\%.
\end{tabular}

Il ressort que le $\mathrm{pH}$ des poissons varie entre 6,04 à 6,60 à bord des bateaux et 6,6 à 7 au niveau de la halle des marées. Cependant, les analyses statistiques ont montré qu'il n'y a aucune différence significative au seuil de 5\% entre les résultats obtenus au niveau des bateaux et à la halle des marées. Par ailleurs, on constate que le $\mathrm{pH}$ des poissons augmente légèrement à la halle des marées. L'Azote Basique Volatil Total (ABVT) dans un produit est lié à la dégradation des protéines contenues dans ce produit. On constate que les teneurs varient d'un échantillon à l'autre au sein d'un même bateau et d'un bateau à l'autre (Tableau 4). Il en est de même à la halle des marées. Les résultats indiquent que $100 \%$ des échantillons de poissons analysés (soit 50) au niveau des bateaux ont révélé une teneur en ABVT inférieure à la norme (25 mg.N/100 g de chair). Par contre, les teneurs en ABVT obtenues au niveau des bateaux ne sont pas significativement différentes au seuil de $5 \%$ aux teneurs obtenues à la halle des marées.

\section{DISCUSSION}

L'absence des salmonelles dans les échantillons analysés peut s'expliquer par le fait qu'il y existe une compétition entre ce germe et les autres retrouvés au niveau des poissons testés. Les travaux de N'diaye (1998) confirment ce résultat. Les salmonelles étant responsables de toxi-infection alimentaire à la suite de l'ingestion des aliments contaminés, une autre raison de leur absence dans les échantillons de poissons analysés serait due aux méthodes de recherches simplifiées. La recherche des salmonelles par la méthode classique peut être négative, alors que l'échantillon en renferme. L'absence des 
Staphylocoques présumés pathogènes dans les échantillons analysés s'expliquerait par le fait que ces poissons ne sont pas trop manipulés à bord des navires par des ouvriers du fait qu'il s'agit des denrées congelées dans la calle ou misses sous glace dans des caisses isothermes. On peut déduire que la glace utilisée pour la conservation de ces poissons à bord des navires n'est pas contaminée par ce germe. De même, selon Borner (2000), les Staphylococcus aureus sont des germes ubiquistes largement répandus dans la nature ; mais la principale source de contamination est l'homme qui héberge les germes au niveau de la peau, des cheveux et de la bouche. Le niveau de contamination élevé des poissons par les GAM observé à la halle des marrées est supérieur à celui observé par N'diaye (1998). Les Germes Aérobies Mésophiles (GAM) assimilés à la flore totale dans un produit alimentaire reflètent la qualité microbiologique générale du produit et permet d'en suivre l'évolution. La flore totale peut donner une indication sur l'état de décomposition du produit et constitue de ce fait un indice de la qualité sanitaire (Anihouvi et al., 2009). Au cours des traitements technologiques, le dénombrement de la flore totale permet de juger de l'incidence des diverses opérations (Borner, 2000). Selon Djinou (2001), les GAM sont des germes «test d'hygiène » du fait qu'ils rendent compte de l'hygiène générale ou d'une conservation inefficace. La forte contamination des Coliformes thermotolérants observée au niveau des poissons pourrait s'expliquer par le manque de la bonne pratique d'hygiène observée souvent à la halle des marées et, d'autre part, par la rupture de la chaîne de froid. La forte contamination des poissons par les Coliformes thermotolérants peut constituer un problème de santé publique dans la mesure où ces germes peuvent être des bactéries de report de contamination (Huss, 1995) qui peuvent se révéler parfois très pathogènes, et qui peuvent résister dans les poissons tout en constituant un maillon de la chaîne alimentaire de ces derniers. Une autre raison du risque sanitaire lié aux Coliformes thermo-tolérants est la production de l'histamine, une amine biogène résistante à la chaleur et toxique pour l'homme (Sitti, 2001). Selon Borner (2000), les Coliformes thermo-tolérants constituent des germes de contamination fécale et sont donc des indicateurs des mauvaises conditions d'hygiène lors de la manipulation des denrées. Les résultats obtenus dans le cadre du dénombrement des spores d'anaérobies sulfito-réducteurs (ASR) sont contraires à ceux obtenus par Ouattara (1986) et Knockart (2002). Par contre, ces résultats sont supérieurs à ceux obtenus par N'diaye (1998).

Les résultats observés dans le cadre des caractéristiques physico-chimiques des poissons analysés ont montré que les valeurs $\mathrm{du} \mathrm{pH}$ des poissons sont proches de celles que doivent avoir un poisson mort bien conservé. Selon Love (1980), après la mort du poisson, le $\mathrm{pH}$ initial varie de 5,4 à 7,2. Toutefois, le $\mathrm{pH}$ du muscle du poisson vivant est proche de la neutralité, mais il diminue normalement pendant le premier jour de la mort en raison de la formation d'acide lactique en anaérobiose, puis se stabilise et augmente légèrement par la suite de l'accumulation des composés basiques (Huss, 1995). Par ailleurs, l'augmentation de la valeur du $\mathrm{pH}$ observée à la halle des marées serait due à l'accumulation des composés basiques par les poissons. Les résultats observés dans le cadre de l'évaluation de la teneur en ABVT des poissons analysés ont montré que les valeurs de cette dernière obtenues à la halle des marées sont supérieures à celles obtenues au niveau des bateaux, d'où une diminution de l'état de fraîcheur du poisson. Cette situation pourrait s'expliquer par le non respect de la chaîne de froid ainsi que le développement des microorganismes dans les poissons. Les teneurs élevées en ABVT (25,03 et $27.76 \mathrm{mg}$ $\mathrm{N} / 100 \mathrm{~g}$ de chair) à la halle des marées sont 
presque les mêmes que celles rapportées par Azibé (1991) qui a constaté une teneur de 28,05 mg N/kg sur un des cent échantillons de filets de poissons congelés d'une part, et celles obtenues par Zamma et Séwadé (2010) où les teneurs observées sont comprises entre 25,0 et $26,7 \mathrm{mg} \mathrm{N} / \mathrm{kg}$.

\section{Conclusion}

Le Bénin dispose d'une halle des marées qui se charge de l'accueil et de la distribution des poissons issus de la pêche industrielle. Au vu de l'évaluation de la qualité de ces poissons, on peut dire que la capture et la commercialisation des poissons connaissent des difficultés liées principalement au manque de bonnes pratiques d'hygiène et de conservation à la halle des marées. La flore microbienne des poissons ainsi que les teneurs en ABVT sont plus élevées à la halle des marées qu'au niveau des bateaux. Cette situation témoigne une fois de plus des conditions d'hygiène peu satisfaisantes et de stockages aussi bien au niveau des produits eux-mêmes, qu'au niveau du matériel utilisé.

\section{RÉFÉRENCES}

Anihouvi VB, Sakyi-Dawson E, Ayernor G.S, Hounhouigan JD. 2009. Biochemical changes and aroma development during the spontaneous fermentation of cassava fish into lanhouin and their influence on product acceptability. Journal of Aquatic Food Product Technology, 18: 370-384.

Azibé M. 1991. Contribution à l'étude de la qualité parasitologique, bactériologique et chimique des filets de poissons congelés produits au Sénégal. Thèse Méd. Vét, Dakar, 19, 96p

Bornert G. 2000. Intérêt et limites des analyses microbiologiques des denrées dans une stratégie de maîtrise de la sécurité des aliments : cas de la restauration collective. Bulletin Vét. France, 153: 433-442.
Cohen N, Ennaji H. 2006. The bacterial quality of red meat and offal in Casablanca (Morocco). Mol. Nutr. Food Res., 50: 557- 562.

Djinou HPAB. 2001. Etude de la qualité microbiologique du poisson fumé artisanalement en Côte d'Ivoire et destiné à l'exportation. Thèse : Méd. Vét : Dakar; 23.

FAO. 2006. Profil des pêches et de l'aquaculture par pays ; vue générale du secteur des pêches nationales FAO/FIR/CP/BEN p 42.

Gbaguidi P, Fiogbé I. 2006. Rapport national sur la pêche au Bénin.

Gram L. 1987. Spoilage of three Senegalese fish species stored in ice and at ambient temperature. Paper presented at SEAFOOD 2000 in Halifax, Canada.

Huss H. 1995. Safety of Seafoods. FAO: Rome; 63p.

Kaferstein F, Motarjemi K. 1997. Foodborne disease control: a transnational challenge. Emer. Infect. Disease, 3: 603-610.

Knockart C. 2002. Le Fumage du Poisson (7è édn). Ifremer: Nantes; 174p.

Lalèyè P. 1995. Ecologie comparée de deux espèces de chrysichtys, poissons siluriformes (Claroteidae) du complexe lagunaire lac Nokoué, lagune de PortoNovo au Bénin. Mémoire de thèse, Belgique, p. 152.

Liston J. 1992. Bacterial spoilage of seafood. Quality Assurance in the Fish Industry. Proceedings of an International Conference, Copenhagen, Denmark, August 1992. Elsevier, 2: 93-105.

Love RM. 1980. Biochemical dynamics and the quality of fresh and frozen fish. In Fish Processing Technology (2nd edn), Hall GM (ed.). Blackie Academic \& Professional (Chapman \& Hall): London; 1-26.

N'diaye A. 1998. Contribution à l'étude de l'évolution de la qualité bactériologique des produits de la pêche destinés à 
l'exportation en 1998 et 1997 Thèse Méd. Vét., Dakar 17, 73p.

Ouattara B. 1986. Etude de la qualité bactériogique des filets de poisons congelés. Thèse : Méd. Vét : Dakar ; 20.

Sitti AH. 2001. Contribution à l'étude de l'évolution de la qualité bactériologique des produits de la pêche de 1997-2000. Thèse : Méd. Vét : Dakar ; 17.

Sylla K. 2000. Contribution à l'étude comparée des conditions de réception, de stockage et de préparation des denrées alimentaires d'origine animale en restauration collective: cas particulier des restaurants du Centre des Euvres Universitaires de Dakar (COUD)Sénégal. Thèse Méd. Vét.: Dakar.

Zamma E, Sewadé F. 2010. Evaluation de la teneur en Azote Basique Volatile Totale dans l'un des poissons les plus consommés au Bénin : cas des chinchards. Mémoire de Licence en Biotechnologie Alimentaire, GAZA Formation, Cotonou, 54p. 